\title{
Monitoramento metacognitivo de adultos em processo de alfabetização em tarefas de memória, processamento e inteligência geral
}

\author{
Márcia Akemi Fujie ${ }^{1}$ \\ Patrícia Waltz Schelini ${ }^{1}$ \\ ${ }^{1}$ Universidade Federal de São Carlos, SP, Brasil
}

\begin{abstract}
Resumo
A autorregulação refere-se aos pensamentos, sentimentos e ações que são planejados e ciclicamente adaptados para o alcance dos objetivos traçados pelo indivíduo. Dentro dessa totalidade, encontra-se o monitoramento metacognitivo, habilidade pela qual o sujeito "acompanha" e avalia sua própria cognição. O presente estudo teve como objetivo investigar o monitoramento de adultos em processo de alfabetização por meio do julgamento, entendido como uma medida do monitoramento metacognitivo, sobre o desempenho em tarefas cognitivas que avaliam o fator geral de inteligência, a velocidade de processamento e a memória de curto prazo. Também foi investigada a existência de diferenças nas relações entre desempenhos reais e estimados e em quais tarefas cognitivas o desempenho real mais se relaciona ao desempenho estimado. Participaram do estudo 34 alunos de um programa de alfabetização para adultos, de ambos os gêneros e com idade entre 40 e 60 anos. Os materiais utilizados para a coleta de dados foram: Entrevista Inicial (EI), Teste R-1 e os subtestes Código, Procurar Símbolos e Dígitos da Escala Wechsler de Inteligência para Adultos III (WAIS III), e Registro de Julgamento de 0 a 100 . Os resultados indicaram que os escores reais dos participantes foram mais baixos quando comparados com sujeitos de maior escolaridade das amostras normativas dos instrumentos. A partir das correlações de Pearson, observou-se correlação significativa entre o desempenho real e o julgamento do desempenho no subteste Códigos.
\end{abstract}

Palavras-chave: Alfabetização; Avaliação; Metacognição.

\section{Metacognitive monitoring in adults in a literacy program in memory, processing and general factor of intelligence tasks}

\begin{abstract}
Self-regulation refers to thoughts, feelings and actions that are planned and cyclically adapted so as to make the individual reach their goals. Within the self-regulation is the metacognitive monitoring, the ability with which the individual follows and evaluates their own cognition. The present study aimed to investigate the metacognitive monitoring of illiterate adults using the metacognitive judgement, a measure of the metacognitive monitoring, in cognitive tasks that evaluate the general factor of intelligence, processing speed and short-term memory. The relation between the real performance and the estimated performance was also investigated, in which cognitive task the real performance related more to the estimated performance. The participants of the study included 34 students in a literacy program for adults, from both genders, and age between 40 and 60 years old. The instruments utilized were: Initial Interview, R-1 Test and subtests Digit Symbol-Coding, Symbol Search and Digit Span from the Wechsler Adult Intelligence Scale III (WAIS III), and Judgement Register from 0 to 100. Results indicated that the real performance of the subjects were lower when compared to subjects with a greater level of education. According to the Pearson product-moment correlation coefficient, there was a significant correlation between the real performance and the estimated performance in the subtest Digit Symbol-Coding.
\end{abstract}

Keywords: Evaluation; Literacy; Metacognition.

\section{Monitoreo metacognitivo de adultos en procedimiento de alfabetización en tareas de memoria, procesamiento e inteligencia general}

\section{Resumen}

La autorregulación se refiere a los pensamientos, sentimientos y acciones que son planificados y cíclicamente adaptados para el logro de los objetivos trazados por el individuo. Dentro de esa totalidad, se encuentra el monitoreo metacognitivo, que es la habilidad por la cual el sujeto "acompaña" y evalúa su propia cognición. El presente estudio tuvo como objetivo investigar el monitoreo de adultos en proceso de alfabetización, a través del juicio, entendido como una medida del monitoreo metacognitivo, sobre el desempeño en tareas cognitivas que evalúan el factor general de inteligencia, la velocidad de procesamiento y la memoria de corto plazo. También se investigó la existencia de diferencias en las relaciones entre desempeños reales y estimados y en cuáles tareas cognitivas el desempeño real más se relaciona con el desempeño estimado. Participaron del estudio 34 alumnos de un programa de alfabetización para adultos, de ambos géneros y con edad entre 40 y 60 años. Los materiales utilizados para la recolección de datos fueron: Entrevista Inicial (EI), Prueba R-1 y los subtestes Código, Buscar Símbolos y Dígitos de la Escala Wechsler de Inteligencia para Adultos III (WAIS III) y Registro de Juicios de 0 a 100. Los resultados indicaron que los puntajes reales de los participantes fueron más bajos cuando comparados con sujetos de mayor escolaridad de las muestras normativas de los instrumentos. A partir de las correlaciones de Pearson, se observó una correlación significativa entre el desempeño real y el juicio del desempeño en el subtest Códigos.

Palabras clave: Alfabetización; Evaluación; Metacognición. 


\section{Introdução}

A autorregulação refere-se aos pensamentos, sentimentos e ações que são planejados e ciclicamente adaptados para o alcance dos objetivos traçados pelo indivíduo. É descrita como cíclica porque o feedback de desempenhos prévios é usado para realizar ajustes durante esforços atuais. Tais ajustes são necessários porque fatores pessoais, comportamentais e ambientais estão constantemente mudando durante o curso da aprendizagem e performance (Zimmerman, 2000).

A aprendizagem autorregulada é composta por uma variedade de interações entre componentes cognitivos, metacognitivos e motivacionais (Zimmerman, 2000). A dimensão cognitiva inclui habilidades necessárias para codificar, memorizar e recordar informação, sendo constituída por três tipos gerais de habilidades de aprendizagem: estratégias cognitivas, estratégias de resolução de problemas e habilidades de pensamento crítico (Schraw, Crippen, \& Hartley, 2006). Já a dimensão metacognitiva, a ser enfatizada no presente estudo, inclui habilidades que possibilitam entender e monitorar os processos cognitivos. Inclui dois principais subcomponentes: o conhecimento da cognição (ou conhecimento metacognitivo) e a regulação da cognição. O primeiro refere-se ao que o indivíduo sabe sobre sua própria cognição e o segundo inclui os componentes de planejamento, monitoramento e avaliação (Schraw, Crippen, \& Hartley, 2006).

Estudos sobre a metacognição ainda são recentes na literatura e foram introduzidos inicialmente por Flavell na década de 70 . O termo refere-se ao conhecimento sobre o próprio conhecimento, o conhecimento sobre a natureza cognoscente das pessoas, a natureza das diferentes tarefas cognitivas e as possíveis estratégias que podem ser aplicadas para a solução dessas, além das habilidades executivas para o monitoramento e regulação das atividades cognitivas (Flavell, 1979).

Flavell e Wellman (1977), no início dos trabalhos sobre metacognição, limitaram-se ao conhecimento dos sujeitos sobre sua própria cognição, como, por exemplo, o que reconheciam sobre sua memória (metamemória) e o que sabiam sobre sua atenção (Flavell \& Wellman, 1977; Jou \& Sperb, 2006). A compreensão da metacognição, com o decorrer dos estudos, foi ampliada para além do conceito de conhecimento metacognitivo, sendo entendida também como a principal responsável por possibilitar a regulação da cognição e, portanto, o monitoramento, controle dos processos cognitivos e uso de estratégias de potencialização da cognição (Jou \& Sperb, 2006).

Assim, partindo-se do conceito de metacognição, é possível conferir um papel ativo ao indivíduo em seu desempenho nas atividades cognitivas, com implicações significativas para o campo escolar e da aprendizagem. A metacognição é imprescindível para auxiliar no direcionamento e redirecionamento das ações cognitivas de maneira efetiva. Um desempenho metacognitivo adequado pode ser útil em outras áreas além da educacional, como em situações de comunicação que contemplem exposição oral de informações, orientação, ou compreensão de conversação oral ou escrita. Além disso, outras tarefas cognitivas como as que abrangem atenção, autocontrole, memória, percepção, raciocínio lógico e resolução de problemas também podem ser potencializadas pelo desempenho metacognitivo (Flavell, Miller, \& Miller, 1993; Zampieri, 2012).

Dentre os diversos componentes e processos metacognitivos, deseja-se destacar, no presente estudo, a relevância do monitoramento metacognitivo na obtenção de conhecimento sobre o próprio desempenho. A partir do monitoramento, o indivíduo é capaz de auto avaliar sua aprendizagem e observar a necessidade ou não de modificar suas estratégias, de forma a obter resultados mais vantajosos. Esta é uma habilidade que permite identificar possíveis dificuldades nos campos da atenção, compreensão, estados afetivos e motivacionais em relação à aprendizagem, criando-se uma relação entre a performance da tarefa e o julgamento do indivíduo sobre seu desempenho (Boruchovitch, 2014; Gutierrez, Schraw, Kuch \& Richmond, 2016; Roebers, Krebs \& Roderer, 2014). Dessa maneira, considerar o papel do monitoramento metacognitivo dentro do estudo da metacognição implica evidenciar a medida de consciência do indivíduo sobre seu desempenho cognitivo (Zampieri, 2012).

Em um estudo que buscou avaliar como o monitoramento metacognitivo foi avaliado em pesquisas nacionais e internacionais nos últimos 10 anos, Schelini, Deffendi, Fujie, Boruchovitch e Freitas (2016) observaram que a maioria dos trabalhos foi composta por amostras de universitários, estudantes de ensino médio e sujeitos com ensino superior completo. Não foram encontrados quaisquer outros estudos que tivessem como população pré-escolares ou adultos analfabetos.

\section{Alfabetização e letramento}

Apesar de distintos, a alfabetização e o letramento são processos inerentes. A alfabetização, apesar de possuir diversos aspectos interpretativos (Haddad \& Siqueira, 2015) e caracterizar-se por uma gama de habilidade, em seu sentido mais abrangente refere-se à aprendizagem da leitura e escrita. O letramento, por sua vez, transcorre de aplicações sociais nas quais a 
leitura e escrita são empregadas em diversos contextos que abrangem compreensão e representação dialética e verbal (Moraes, 2005; Soares, 2011).

Dessa forma, a alfabetização desenvolve-se dentro e a partir de contextos sociais de leitura e escrita, ou seja, através do letramento. Por sua vez, o letramento evolui a partir do contexto e aprendizagem da correlação grafema-fonema (Soares, 2007). Juntamente com o conceito estabelecido pelo Ministério da Educação de que o indivíduo alfabetizado não apenas domina o princípio do ler e o escrever e/ou poucos conceitos numéricos, como também se qualifica no uso da linguagem escrita e domínio de conceitos matemáticos em diversos contextos (INEP, 2015), acrescenta-se que a demanda não se concentra apenas nas habilidades de ler e escrever, mas igualmente no que o sujeito é capaz de estabelecer com tais competências (Ribeiro, 2006).

Seguindo a classificação de Ribeiro, Vóvio e Moura (2002), foram adotadas quatro classificações possíveis para a condição de alfabetismo: 1) analfabetismo; 2) alfabetismo nível 1 (indivíduo possui reduzida aquisição de alfabetização e letramento); 3) alfabetismo nível 2 (indivíduo com domínio mínimo de alfabetização e utilização de suas habilidades em contexto social); e 4) alfabetismo nível 3 (indivíduo alcança capacidades de alfabetização e letramento que formam o mínimo especificado pela educação básica).

Entretanto, é importante ressaltar que o conceito de letramento possibilita a criação de um continuum, ou seja, graus de letramento. Não existe um limite para o letramento, uma vez que a sociedade evoluiu e desenvolve novas formas de escrever e estruturas para leitura. Dessa forma, os níveis aqui apresentados não são absolutos ou definitivos, sendo desenvolvidos apenas para facilitar a classificação das habilidades dos participantes.

Levando-se em conta que o Brasil possui a oitava maior população no mundo de adultos analfabetos, cerca de 14 milhões de pessoas (UNESCO, 2014), e diante da relevância do monitoramento metacognitivo e da investigação de estudos nacionais e internacionais sobre o tema, surgiu o interesse em explorá-lo em indivíduos adultos, analfabetos e em processo de escolarização, exatamente pela escassez de estudos com essa específica população. Além disso, a literatura tem demonstrado que a metacognição é elemento essencial para a aprendizagem formal, podendo ser articulado e impulsionado pela educação (Cornoldi, 2010; Jou \& Sperb, 2006). Dessa forma, o conhecimento adquirido por meio dos estudos com esta população fornecerá subsídios relevantes para as instituições de ensino, e poderá ser utilizado na formação e aperfeiçoamento de medidas de avaliação do funcionamento metacognitivo, de forma a ampliar o desenvolvimento dos indivíduos nos âmbitos acadêmicos, sociais e pessoais. A troca de experiência e conhecimento entre as instituições de ensino e o grupo de pesquisadores permitirá a difusão de informações sobre a metacognição, assim como o aumento da compreensão acerca desta capacidade, incentivando práticas voltadas para seu desenvolvimento.

Um levantamento realizado para a presente pesquisa na literatura brasileira e internacional nas bases de dados SciELo, Pepsic, LivRe e Index Psi, sem a especificação de período de tempo, não possibilitou a obtenção de qualquer estudo sobre a metacognição de adultos analfabetos ou em processo de alfabetização, e o mesmo foi constatado no trabalho de Schelini et al. (2016), que analisou as bases PsycInfo, Web of Science e SciELo entre os anos de 2005 e 2015.

\section{Método}

\section{Participantes}

Participaram do estudo 34 adultos, 25 do gênero feminino e 9 do gênero masculino, com idades entre 40 e 60 anos (média de 47,76 anos), sendo 12 analfabetos e 22 alfabetizados nível 1 .

\section{Local}

A coleta foi realizada em uma Escola Municipal de Ensino Fundamental, localizada na cidade de São Paulo, com classes de alunos da Educação de Jovens e Adultos (EJA), sem ruídos ou outras atividades que pudessem interferir no procedimento.

\section{Instrumentos}

1. Entrevista Inicial, utilizada para a obtenção de dados sobre a identificação e escolaridade dos participantes.

2. Teste R-1: Teste Não Verbal de Inteligência, criado por Oliveira (1973) e adaptado por Alves (2002), com o objetivo de avaliar a inteligência de adultos, mais especificamente o fator geral de inteligência. É composto por 40 itens não verbais, apresentados em um caderno, cada página contendo um item diferente, o que evita a interferência de um item sobre o outro e possibilita a maior concentração do examinado. Os itens são constituídos por uma figura que possui uma parte faltando, que deve ser completada por uma das alternativas apresentadas abaixo da mesma. Nos itens iniciais, os objetos mostrados são estímulos concretos, comuns. 
Posteriormente, os itens ficam mais complexos, com o uso de figuras geométricas e inclusão de relações de identidade e analogia, além da ampliação da quantidade de elementos na matriz e relações de soma e subtração (Gottsfritz \& Alves, 2009). As respostas são marcadas em uma folha à parte, permitindo que sua aplicação seja feita de maneira individual ou coletiva, e, no presente estudo, o instrumento foi aplicado individualmente. Este é um teste com estudos que evidenciam sua validade e precisão para uso na população brasileira. No presente estudo, em relação à população analfabeta, $\mathrm{o}$ instrumento foi aplicado individualmente.

3. Escala Wechsler de Inteligência para Adultos III (WAIS-III), elaborada por Wechsler (1997) e adaptada para uso no Brasil por Nascimento (2000), com finalidade de investigar o desempenho intelectual de adolescentes e adultos. É composta por 14 subtestes, agrupados em dois conjuntos: Verbal (subtestes Vocabulário, Semelhanças, Aritmética, Dígitos, Informação, Compreensão e Sequência de Números e Letras) e Execução (subtestes Completar Figuras, Códigos, Cubos, Raciocínio Matricial, Arranjo de Figuras, Procurar Símbolos e Armar Objetos). Os subtestes escolhidos para a presente pesquisa foram Códigos, no qual cada número está associado a um código diferente, e o participante deve copiar cada desenho na sequência numérica apresentada; e Procurar Símbolos, no qual o participante deve buscar determinados símbolos e assinalar "sim" ou "não" caso estejam ou não presentes na sequência apresentada, sendo ambos destinados à avaliação da velocidade de processamento cognitivo; e Dígitos, no qual o participante deve repetir, em voz alta, os números enunciados pelo avaliador na mesma sequência, ou na sequência inversa, com o objetivo de avaliar a memória de curto prazo.

4. Registro de julgamentos de 0 a 100 , consiste em uma folha de registro elaborada pela pesquisadora com base no material proposto por Zampieri (2012), com o objetivo do participante estimar seu desempenho no Teste R-1 e nos subtestes Código, Procurar Símbolos e Dígitos do WAIS-III. Após a realização de cada atividade, a pesquisadora lia a seguinte instrução ao participante: "Pense no teste que acabou de fazer. Indique de 0 a 100, como se fosse uma nota para o que fez", de maneira que o indivíduo realizava, então, um julgamento retrospectivo do seu desempenho.

\section{Procedimento}

O projeto foi aprovado pelo Comitê de Ética em Pesquisas em Seres Humanos (CAAE 48461015.3.0000. 5504), sendo que somente participaram da pesquisa os sujeitos que concordaram com o Termo de Con- sentimento Livre e Esclarecido (TCLE), contendo informações e esclarecimentos sobre o procedimento e objetivos do estudo.

A pesquisadora realizou um primeiro contato com possíveis participantes, de maneira a explicar o objetivo da pesquisa e averiguar o interesse de participação no estudo. Foi, então, combinado um horário de encontro para a coleta de dados. O encontro com cada participante durou cerca de 60 minutos. A sessão foi subdividida da seguinte maneira: 1) explicação da pesquisa, leitura do TCLE e obtenção de dados gerais por meio da Entrevista Inicial; 2) aplicação do Teste R-1 e Registro de Julgamentos; 3) aplicação dos subtestes Código e Registro de Julgamentos; Procurar Símbolos e Registro de Julgamentos; e Dígitos e Registro de Julgamentos.

\section{Análise de dados}

Os dados da entrevista inicial foram avaliados a partir de um roteiro de sistematização dos dados; e na análise do monitoramento metacognitivo, os reais desempenhos (escores brutos) dos participantes no Teste R-1 e subtestes Código, Procurar Símbolos e Dígitos foram correlacionados aos desempenhos estimados pelos participantes. Ainda, foram correlacionados os julgamentos com os escores ponderados dos participantes.

\section{Resultados}

Serão descritos, inicialmente, o desempenho dos participantes no Teste R-1 e subtestes Códigos, Dígitos e Procurar Símbolos da Escala Wechsler de Inteligência para Adultos III (WAIS-III), de acordo com seus escores brutos (Tabela 1). Em seguida, será apresentada a classificação dos participantes no Teste R-1 e subtestes Códigos, Dígitos e Procurar Símbolos de acordo com seus percentis (Tabela 2).

A Tabela 1 indica informações sobre as médias, desvios-padrão e variância relativos ao desempenho (desempenho real) dos 34 participantes nos quatro testes e subtestes aplicados.

Pode-se observar que o teste que apresentou menor média de pontuação foi o subteste Procurar Símbolos $(8,76)$, também tendo o menor desempenho mínimo. Já o teste com a maior média de pontuação foi o subteste Códigos (22,12), seguido pelo Teste R-1 (11,62). Essas médias devem ser interpretadas com muita cautela, uma vez que as pontuações máximas possíveis variam de teste para teste.

A Tabela 2 indica informações sobre as médias, desvios-padrão e variância relativos ao desempenho estimado (julgamento) de 0 a 100 dos 34 participantes nos quatro testes aplicados. 
TABELA 1

Estatística descritiva do desempenho no Teste R-1 e subtestes Códigos, Dígitos e Procurar Símbolos

\begin{tabular}{lcccccc}
\hline & $N$ & Mínimo & Máximo & Média & Desvio Padrão & Variância \\
R-1 & 34 & 2,00 & 22,00 & 11,62 & 4,559 & 20,789 \\
Códigos & 34 & 2,00 & 41,00 & 22,12 & 9,393 & 88,228 \\
Dígitos & 34 & 4,00 & 16,00 & 9,94 & 2,785 & 7,754 \\
Procurar Símbolos & 34 & 0,00 & 18,00 & 8,76 & 4,486 & 20,125 \\
\hline
\end{tabular}

TABELA 2

Estatística descritiva do julgamento de desempenho de 0 a 100 no Teste R-1 e subtestes Códigos, Dígitos e Procurar Símbolos

\begin{tabular}{lcccccc}
\hline & $N$ & Mínimo & Máximo & Média & Desvio Padrão & Variância \\
R-1 & 34 & 0,00 & 100,00 & 27,24 & 30,569 & 934,488 \\
Códigos & 34 & 0,00 & 100,00 & 29,82 & 35,997 & 1295,789 \\
Dígitos & 34 & 0,00 & 100,00 & 24,38 & 29,920 & 895,213 \\
Procurar Símbolos & 34 & 0,00 & 100,00 & 33,76 & 36,895 & 1361,216 \\
\hline
\end{tabular}

Constata-se que todos os testes tiveram como julgamento de desempenho mínimo a nota 0 , e como nota máxima 100. Além disso, o subteste Procurar Símbolos teve a maior média de julgamento de desempenho $(33,76)$, seguido pelo subteste Códigos $(29,82)$ e Teste R-1 $(27,24)$.

Uma vez apresentadas as informações a respeito do desempenho dos participantes no Teste R-1 e subtestes Códigos, Dígitos e Procurar Simbolos, são exibidas a seguir a classificação de resultados a partir dos seus percentis (Tabela 3 e Tabela 4).

TABELA 3

Percentis por Amostra Total no Teste R-1

\begin{tabular}{lcc}
\hline Classificação (percentis) & $N$ & $\%$ \\
Inferior (1-5) & 11 & 32,35 \\
Médio Inferior (10-25) & 18 & 52,95 \\
Médio (30-70) & 5 & 14,70 \\
Médio Superior (75-90) & 0 &, 00 \\
Superior (95) & 0 &, 00 \\
Muito Superior (>99) & 0 &, 00 \\
\hline
\end{tabular}

Nota-se que a maioria dos participantes apresentou desempenho inferior $(32,35 \%)$ ou médio inferior $(52,95 \%)$ no Teste R-1, com nenhum participante atingindo a classificação média superior, superior ou muito superior.

$\mathrm{Na}$ classificação dos participantes pelos percentis nos subtestes Códigos, Dígitos e Procurar Símbolos, nenhum participante obteve os maiores valores (16 a 19), sendo que a maior parte dos desempenhos se concentrou entre os percentis 6 e 10 .

Utilizando-se os valores de assimetria entre -3 e +4 (Kline, 2011) e valores de curtose entre -8 e +8 (Kline, 2011) para uma distribuição normal, pôde-se observar que, em relação ao Teste R-1, as respostas dos 34 participantes apresentaram uma distribuição que tendeu à normalidade (Assimetria $=0,720$; Curtose $=-0,105)$, assim como nos subtestes Códigos (Assimetria $=-0,102$; Curtose $=-0,155)$, Dígitos (Assimetria $=-0,115$; Curtose $=-0,193$ ) e Procurar Símbolos (Assimetria =0,143; Curtose $=0,018$ ). Dessa maneira, foram utilizados testes estatísticos paramétricos para realizar análises correlacionais entre os escores brutos e julgamentos de desempenho, e entre os percentis e julgamentos de desempenho.

TABELA 4

Percentis por Amostra Total nos subtestes Códigos, Dígitos e Procurar Símbolos

\begin{tabular}{|c|c|c|c|c|c|c|}
\hline $\begin{array}{l}\text { Classificação } \\
\text { (percentis) }\end{array}$ & $\begin{array}{l}\text { Códigos } \\
\text { (n) }\end{array}$ & $\%$ & $\begin{array}{l}\text { Dígitos } \\
\text { (n) }\end{array}$ & $\%$ & $\begin{array}{c}\text { Procurar Símbolos } \\
\text { (n) }\end{array}$ & $\%$ \\
\hline $1-5$ & 2 & 5,80 & 3 & 8,80 & 4 & 11,80 \\
\hline $6-10$ & 31 & 91,30 & 22 & 64,70 & 30 & 88,20 \\
\hline $11-15$ & 1 & 2,90 & 9 & 26,50 & 0 &, 00 \\
\hline $16-19$ & 0 &, 00 & 0 &, 00 & 0 & ,00 \\
\hline
\end{tabular}


TABELA 5

Correlações de Pearson entre os escores brutos do Teste R-1 e os subtestes Códigos, Dígitos e Procurar Símbolos e seus respectivos julgamentos de 0 a 100

\begin{tabular}{|c|c|c|c|c|}
\hline & $R-1$ & Códigos & Dígitos & Procurar Símbolos \\
\hline Julgamento R-1 & 0,019 & & & \\
\hline Julgamento Códigos & & $0,352 *$ & & \\
\hline Julgamento Dígitos & & & 0,224 & \\
\hline Julgamento Procurar Símbolos & & & & 0,107 \\
\hline
\end{tabular}

* Correlação de Pearson significativa a 0,05 .

TABELA 6

Correlações de Pearson entre percentis do Teste R-1 e os subtestes Códigos, Dígitos e Procurar Símbolos e seus respectivos julgamentos de 0 a 100

\begin{tabular}{|c|c|c|c|c|}
\hline & $R-1$ & Códigos & Dígitos & Procurar Símbolos \\
\hline Julgamento R-1 & $-0,048$ & & & \\
\hline Julgamento Códigos & & 0,335 & & \\
\hline Julgamento Dígitos & & & 0,224 & \\
\hline Julgamento Procurar Símbolos & & & & $-0,003$ \\
\hline
\end{tabular}

A Tabela 5 apresenta as correlações de Pearson entre os escores totais brutos e os julgamentos de desempenho dos participantes no Teste R-1 e subtestes Códigos, Dígitos e Procurar Símbolos.

Considerando as correlações estabelecidas entre os escores brutos do Teste R-1 e os subtestes Códigos, Dígitos e Procurar Símbolos do WAIS III com os julgamentos dos participantes em relação ao seu desempenho em cada atividade, pode-se observar que houve correlação significativa, porém de moderada a fraca, entre o subteste Códigos e os julgamentos $(r=0,352)$, sendo que todas as outras correlações foram de fraca magnitude e não significativas. Os níveis de associação utilizados foram: alta $r>0,50$, moderada $r=0,35-0.50$ e fraca $r \leq 0,34$ (Dancey \& Reidy, 2013).

A Tabela 6 apresenta as correlações de Pearson entre os percentis dos participantes no Teste R-1 e subtestes Códigos, Dígitos e Procurar Símbolos com seus julgamentos de desempenho nos mesmos.

É possível observar que não houve correlações significativas entre os percentis e os julgamentos de desempenho dos participantes, sendo todas de fraca magnitude (Dancey \& Reidy, 2013).

\section{Discussão e Conclusão}

O objetivo do presente estudo foi investigar o monitoramento metacognitivo de adultos em processo de alfabetização utilizando-se o julgamento (estimativa) sobre o desempenho em tarefas cognitivas. A partir dos escores brutos, percentis e julgamentos, realizaram-se análises de maneira a correlacionar os desempenhos reais e estimados dos participantes, de forma a verificar a existência de correlações significativas entre os resultados.

Primeiramente, é possível observar que os escores totais brutos e percentis obtidos pelos participantes nas medidas psicológicas foram baixos. Este resultado vai ao encontro de muitos estudos que têm demonstrado diferenças significativas de escores entre grupos com menor e maior escolaridade em testes que avaliam a memória de curto prazo (Coelho et al., 2012) e velocidade de processamento (Teixeira-Fabrício et al., 2012), assim como o desempenho no Teste R-1 (Gottsfritz \& Alves, 2009).

A análise da correlação de Pearson entre o escore bruto do desempenho real e julgamento de desempenho apresentou níveis de correlação de baixa magnitude, sendo significativa apenas a correlação entre o subteste Códigos e seu julgamento, apesar de ter sido considerada de magnitude de moderada a fraca. Uma possível explicação para esse resultado é a maior facilidade deste subteste em relação às outras atividades, pois é uma tarefa de baixa complexidade e que tem a velocidade de realização como um fator determinante. No subeste Códigos são apresentadas sete linhas contendo vinte números cada e o sujeito deve desenhar o código (figura) correspondente a cada número durante 120 segundos. Assim, é possível que 
o participante estime seu desempenho pelas linhas que não conseguiu fazer, o que pode ser um fator norteador de estimativas mais próximas do desempenho real.

Os resultados indicam que os participantes tenderam a avaliar seu próprio desempenho como baixo, considerando-se que a nota máxima possível de julgamento de desempenho seria 100 e as médias dos valores de julgamentos foram 27,24 para o Teste R-1, 29,82 para o subteste Códigos, 24,38 para Dígitos e 33,76 para Procurar Símbolos, sendo que o desempenho real nas atividades também tendeu a ser abaixo da média, considerando-se os percentis. Além disso, o desempenho real correlacionado ao desempenho estimado não originou a correlações sequer moderadas, indicando que os participantes tenderam a subestimar ou superestimar seu próprio desempenho, resultado já demonstrado em muitos estudos sobre julgamentos de memória (Benjamin, Bjork, \& Schwartz, 1998; Finn, 2008; Koriat, 1997).

A acurácia do julgamento varia de acordo com diferentes fatores que influenciam o monitoramento metacognitivo (Kessel et al., 2014), como a dificuldade da tarefa e o tempo transcorrido após a realização da tarefa e o julgamento (Kessel et al., 2014; Schraw, Dunkle, Bendixen, \& Roedel, 1995), o que pode fundamentar a correlação significativa entre o desempenho real e o julgamento de desempenho no subteste Códigos, considerado uma das atividades de maior facilidade. Adicionalmente, esses dados sugerem uma dificuldade de monitoramento de desempenho, assim como uma dificuldade na realização de tarefas cognitivas que procuram avaliar velocidade de processamento, memória de curto prazo e inteligência geral, observado pelos baixos escores obtidos no desempenho real dos participantes. Os resultados fortalecem as evidências de que uma melhor atuação nas atividades, em geral, está associada a um melhor monitoramento metacognitivo (Son \& Schwartz, 2005; Zampieri \& Schelini, 2013b).

O campo da metacognição e, mais especificamente, o monitoramento metacognitivo, é recente e escasso de estudos. Além de ser um tema pouco explorado, também não existem muitos estudos sobre a população escolhida, tornando-se mais complicada ainda a investigação proposta. Até o momento, o presente estudo foi o primeiro no Brasil a ter como objeto de pesquisa adultos analfabetos e em processo de alfabetização e seu monitoramento metacognitivo; dessa maneira, é inevitável que existam certas lacunas em sua realização, como a falta de coincidência entre a pontuação máxima possível nos testes aplicados (Teste R-1, 0 a 40; Códigos, 0 a 133; Dígitos, 0 a 30; e Procurar Símbolos, 0 a 60) e na técnica para avaliação do julgamento ( 0 a 100$)$, no que se refere à tentativa de compreensão de que os participantes tenderam a subestimar ou superestimar seus desempenhos.

Os resultados demonstram como adultos analfabetos e em processo da alfabetização apresentam baixos resultados reais nos testes. Além disso, demonstram baixa acurácia na avaliação do próprio desempenho, ocorrendo correlação significativa entre o desempenho real e julgamento de desempenho em apenas um subteste.

É possível que esses resultados apontem os impactos da falta da educação formal, além da dificuldade na realização de atividades cognitivas e, por consequência, em tarefas do dia-a-dia. É necessário que novas pesquisas sobre o tema e com a mesma população sejam realizados, com maior número de participantes e aplicação de outros testes que avaliem mais aspectos da cognição. Os resultados aqui obtidos podem embasar novas políticas públicas de educação, de forma a aumentar o investimento e o incentivo à entrada de adultos analfabetos em instituições de ensino.

\section{Referências}

Alves, I. C. B. (2002). R-1: Teste Não Verbal de Inteligência-Manual. São Paulo: Vetor.

Benjamin, A. S., Bjork, R. A., \& Schwartz, B. L. (1998). The mis-measure of memory: When retrieval fluency is misleading as a meta- mnemonic index. Journal of Experimental Psychology: General, 127, 55-68. https://doi. org/10.1037/0096-3445.127.1.55

Boruchovitch, E. (2014). Autorregulação da aprendizagem: contribuições da psicologia educacional para a formação de professores. Psicologia Escolar e Educacional, 18(3), 401-409. https://doi.org/10.1590/2175-3539/2014/ 0183759

Brasil. (2015). Instituto Nacional de Estudos e Pesquisas Educacionais Anisio Teixeira (Inep). Plano Nacional de Educação PNE 2014-2024. Brasília, DF.

Coelho, F. G. M., Vital, T. M., Novais, I. de P., Costa, G. de A., Stella, F., \& Santos-Galduroz, R. F. (2012). Desempenho cognitivo em diferentes níveis de escolaridade de adultos e idosos ativos. Revista Brasileira de Geriatria e Gerontologia, 15(1), 7-15. https://doi.org/10.1590/S1809-98232012000100002 
Cornoldi, C. (2010). Metacognition, intelligence, and academic performance. Em H.S. Waters e W. Schneider. Metacognition, strategy use, and instructions (pp. 257-277). Nova Iorque: The Guilford Press.

Dancey, C. P. \& Reidy, J. (2013). Estatística sem matemática para psicologia. Porto Alegre: Artmed.

Finn, B. (2008). Framing effects on metacognitive monitoring and control. Memory \& Cognition, 36(4), 813-821. https://doi.org/10.3758/MC.36.4.813

Flavell, J. H. \& Wellman, H. M. (1977). Metamemory. In R. V. Kail \& J. W. Hagen (Orgs.). Perspectives on the development of memory and cognition (3-33). Hillsdale, NJ: Erlbaum.

Flavell, J. H. (1979). Metacognition and cognitive monitoring: a new area of cognitive-developmental inquiry. American Psychologist, 34(10), 906-911. https://doi.org/10.1037/0003-066X.34.10.906

Flavell, J. H. (1999). Cognitive development: Children's knowledge about the mind. Annual Review of Psychology, 50, 21-45. https://doi.org/10.1146/annurev.psych.50.1.21

Flavell, J.H., Miller, P.H., \& Miller, S.A. (1993). Cognitive development (3ํe ed.). New Jersey: Prentice Hall.

Gotsfritz, M. O. \& Alves, I. C. B. (2009). Normas do teste de inteligência não verbal R-1 para adultos não alfabetizados. Interação em Psicologia, 13(1), 59-68. https://doi.org/10.5380/psi.v13i1.15856

Gutierrez, A. P., Schraw, G., Kuch, F., \& Richmond, A. S. (2016). A two-process model of metacognitive monitoring: Evidence for general accuracy and error factors. Learning and Instruction, 44, 1-10. https://doi.org/10.1016/j. learninstruc.2016.02.006

Haddad, S. \& Siqueira, F. (2015). Analfabetismo entre jovens e adultos no Brasil. Revista Brasileira de Alfabetização - ABAlf, 1(2), 88-110.

Jou, G. I., \& Sperb, T.M. (2006). A metacognição como estratégia reguladora da aprendizagem. Psicologia: Reflexão e Crítica, 19(2), 177-185. https://doi.org/10.1590/s0102-79722006000200003

Kessel, R., Gecht, J., Forkmann, T., Drueke, B., Gauggel, S., \& Mainz, V. (2014). Metacognitive monitoring of attention performance and its influencing factors. Psychological Research, 78(4), 597-607. https://doi.org/10.1007/s00426013-0511-y

Kline, R. B. (2011). Principles and practice of structural equation modeling (3를. ed. New York: Guilford Press.

Koriat, A. (1997). Monitoring one's own knowledge during study: A cue-utilization approach to judgments of learning. Journal of Experimental Psychology: General, 126(4), 349-370. https://doi.org/10.1037/0096-3445.126. 4.349

Moraes, M. G. (2005). Alfabetização - leitura do mundo, leitura da palavra - e letramento: algumas aproximações. Revista de Ciências Humanas, Universidade Regional Integrada do Alto Uruguai e das Missões, 7(7), 1-11.

Nascimento, E. (2000). Adaptação da Terceira edição da escala Wechsler de inteligência para adultos (WAIS-III) para uso no contexto brasileiro. Tese de doutorado, Universidade de Brasília, Brasília, DF, Brasil.

Oliveira, R. (1973). R-1: Teste Não Verbal de Inteligência - Manual. São Paulo: Vetor.

Organização das Nações Unidas para a Educação, a Ciência e a Cultura. (2014). Ensinar e aprender: alcançar a qualidade para todos. Disponível em: http://unesdoc.unesco.org/images/0022/002256/225654por.pdf

Ribeiro, V. M. (2006). Analfabetismo e analfabetismo funcional no Brasil. Boletim INAF (São Paulo, Instituto Paulo Montenegro), jul-ago.

Ribeiro, V. M., Vóvio, C. L., \& Moura, M. P. (2002). Letramento no Brasil: alguns resultados do indicador nacional de alfabetismo funcional. Educação \& Sociedade, 23(81), 49-70. http://dx.doi.org/10.1590/S0101-73302002008100004

Roebers, C. M., Krebs, S. S., \& Roderer, T. (2014). Metacognitive monitoring and control in elementary school children: Their interrelations and their role for test performance. Learning and Individual Differences, 29, 141-149. https://doi.org/10.1016/j.lindif.2012.12.003

Schelini, P. W., Deffendi, L. T., Fujie, M. A., Boruchovitch, E. \& Freitas, M. F. R. L. (2016). Avaliação do monitoramento metacognitivo: análise da produção científica. Avaliação Psicológica, 15(spe), 57-65. https://doi.org/10.15689/ ap.2016.15ee.06

Schraw, G., Crippen, K. J., \& Hartley, K. (2006). Promoting self-regulation in Science education: Metacognition as part of a broader perspective on learning. Research in Science Education, 36, 111-139. https://doi.org/10.1007/ s11165-005-3917-8

Schraw, G., Dunkle, M. E., Bendixen, L. D., \& Roedel, T. D. (1995). Does a general monitoring skill exist? Journal of Educational Psychology, 87(3), 433-444. https://doi.org/10.1037/0022-0663.87.3.433

Soares, M. B. (2007). Letramento: um tema em três gêneros (4⿳亠丷a ed.). São Paulo: Autêntica Editora.

Soares, M. B. (2011). Alfabetização e letramento (6ª ed.). São Paulo: Contexto.

Teixeira-Fabrício, A., Lima-Silva, T. B., Kissaki, P. T., Vieira, M. G., Ordonez, T. N., Oliveira, T. B., Aramaki, F. O., Souza, P. F., \& Yassuda, M. S. (2012). Treino cognitivo em adultos maduros e idosos: impacto de estratégias segundo faixas de escolaridade. Psico-USF, 17(1), 85-95. https://doi.org/10.1590/S1413-82712012000100010

Wechsler, D. (1997). Wechsler Adult Intelligence Scale-Third Edition (WAIS-III). Admnistrations and Scoring Manual. San Antonio, Texas: Psychological Corporation.

Zampieri, M. \& Schelini, P. W. (2013a). O uso de medidas intelectuais na análise do monitoramento metacognitivo de crianças. Psicologia: Teoria e Pesquisa, 29, 81-88. https://doi.org/10.1590/S0102-37722013000200007 
Zampieri, M. \& Schelini, P. W. (2013b). Monitoramento metacognitivo de crianças de acordo com o nível de desempenho em medidas de capacidade intelectual. Psico, 44, 280-287.

Zampieri, M. (2012). Investigação do monitoramento metacognitivo de crianças diante de medidas de capacidades intelectuais. Dissertação de mestrado, Universidade Federal de São Carlos, São Carlos, SP, Brasil.

Zimmerman, B. J. (2000). Attaining self-regulation: A social cognitive perspective. In M. Boekaerts, P. R. Pintrich \& M. Zeidner (Orgs.). Handbook of self-regulation (pp. 13-39). San Diego, CA: Academic Press. https://doi.org/10.1016/ B978-012109890-2/50031-7

Dados dos autores:

Márcia Akemi Fujie - Doutoranda, Universidade Federal de São Carlos.

Patrícia Waltz Schelini - Doutora, Universidade Federal de São Carlos.

Endereço para correspondência:

Márcia Akemi Fujie

UFSCar - Departamento de Pós-Graduação em Psicologia

Rodovia Washington Luís, km 235 - Caixa Postal 676

13565-905 São Carlos, SP, Brasil

$<$ marcia.fujie@gmail.com>

Recebido em: 11.07 .2017

Aceito em: 30.10.2017 